\title{
Homocoupling of terminal alkynes on calcite (10.4)
}

\author{
Antje Richter ${ }^{\mathrm{a}}$, Manuel Vilas-Varela ${ }^{\mathrm{b}}$, Diego Peña ${ }^{\mathrm{b}, *}$, Ralf Bechstein ${ }^{\mathrm{a}}$, Angelika Kühnle ${ }^{\mathrm{a}, *}$ \\ a Institute of Physical Chemistry, Johannes Gutenberg University Mainz, Duesbergweg 10-14, Mainz 55099, Germany

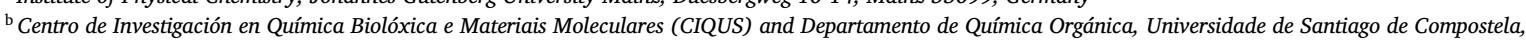 \\ Santiago de Compostela 15782, Spain
}

\section{A R T I C L E I N F O}

\section{Keywords:}

On-surface synthesis

Glaser coupling

Terminal alkynes

Atomic force microscopy

Bulk insulator

Molecular electronics

\begin{abstract}
A B S T R A C T
On-surface synthesis has been identified as highly versatile strategy to prepare molecular structures on surfaces with single-atom precision. Inspired by the classical Glaser coupling, homocoupling of terminal alkynes has attracted great attention for on-surface synthesis. This coupling is known for providing a rigid and linear linkage, which is highly interesting for the synthesis of molecular wires. For molecular wire formation, non-conductive substrates are needed for electronic decoupling. So far, however, coupling of terminal alkynes has not been performed on a bulk insulator surface. Here, we present an atomic force microscopy study, indicating that $4,4^{\prime \prime}$ diethynyl-[1,1':4',1"-terphenyl]-2', 5'-dicarboxylic acid undergoes dimerization by homocoupling of the terminal alkyne moiety on the (10.4) surface of the bulk insulator calcite. Our results suggest that upon low-temperature deposition monomers are found on the surface, which can be dimerized by annealing the sample. When following a high-temperature deposition protocol, the dimerization appears to happen already in the crucible and dimers are deposited directly. Our work, thus, indicates that homocoupling of terminal alkynes can also be performed on a non-metallic surface, in line with a recent theoretical study that suggest the role of the surface being the constrain of the chemical motion rather than contributing in terms of electron transfer.
\end{abstract}

(C) 2017 Elsevier B.V. All rights reserved.

\section{Introduction}

The bottom-up synthesis of functional molecular structure has attracted great attention ever since Richard Feynman gave his inspiring speech in 1959, claiming that "There is plenty of room at the bottom" [1]. In the spirit of this idea, it was Karl-Heinz Rieder and coworkers who have demonstrated that all steps of a chemical reaction can be performed using the tip of a scanning tunneling microscope [2]. In this pivotal work, the classical Ullmann coupling of iodobenzene has been induced on a copper (111) surface, demonstrating molecular engineering at the single molecule level. Nowadays, the field of on-surface synthesis is a highly active research area, which holds great promises for the fabrication of tailor-made functional devices at surfaces [3-6]. Ullmann-like coupling has been used as a popular linking motif on various metal surfaces [7-12]. To enlarge the possible linking schemes for on-surface reactions, Glaser-inspired homocoupling [13] has been explored as a route to couple terminal alkynes and construct rigid linear linkers [14,15]. This reaction has first been presented using 1,3,5triethynyl-benzene on a silver (111) surface [16], but is now well studied on a wide range of conductive surfaces [17-20]. Interestingly, in one of these studies, the dimerization of the 1,4-diethynylbenzene precursor molecules in the crucible has been reported, which has been induced by annealing the crucible to $105{ }^{\circ} \mathrm{C}$ during molecule deposition [17]. Moreover, the formation of unwanted side products upon linkage of a linear diethynyl terphenyl on flat terraces has been addressed by using the alignment of the molecules at the step edges on a vicinal $\mathrm{Ag}(877)$ surface [21]. Also, photochemical activation as an alternative route to annealing has been successfully presented [19]. A detailed theoretical study has been devoted to unravel the molecular reaction mechanism of 1,3,5-triethynyl-benzene linking on $\mathrm{Ag}(111)$ and to uncover the role of the surface, which is explained by constraining the molecular motion rather than contributing chemically in terms of electron transfer [22].

In the view of this growing body of research on metal surfaces it is interesting to raise the question as to whether homocoupling of terminal alkynes can also be achieved on a bulk insulator surface. For this purpose, we investigate $4,4^{\prime \prime}$-diethynyl-[1,1': $4^{\prime}, 1^{\prime \prime}$-terphenyl $]-2^{\prime}, 5^{\prime}$ dicarboxylic acid (DETDCA, Fig. 1a) on the (10.4) cleavage plane of calcite (Fig. 1b) using dynamic atomic force microscopy (AFM) operated in ultrahigh vacuum (UHV).

The molecule is equipped with two ethynyl groups, which are intended for on-surface homocoupling. Additionally, the molecule is also equipped with two carboxylic acid functionalities, which have previously proven to act as suitable anchors on the calcite (10.4) surface and prevent the desorption at elevated temperatures. Density-functional theory calculations have suggested an interaction of the carbonyl groups

\footnotetext{
* Corresponding authors.

E-mail addresses: diego.pena@usc.es (D. Peña), angelika.kuehnle@uni-bielefeld.de, kuehnle@uni-mainz.de (A. Kühnle).
} 
(a)

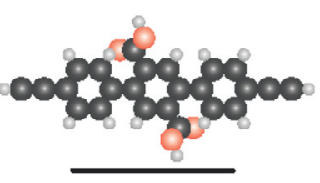

$1 \mathrm{~nm}$ (b)

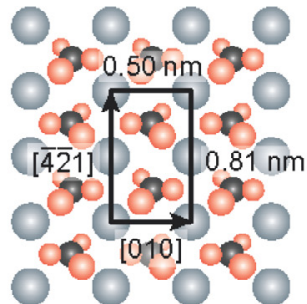

calcium

carbon

oxygen

hydrogen

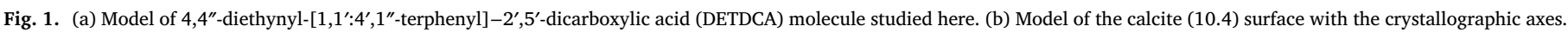
The surface unit cell is marked by the black rectangle. The scale bar applies to both subsets.<smiles>CCOC(=O)c1cc(O)c(C(=O)OCC)cc1O</smiles><smiles>CCOCCN(C)CC</smiles>
1<smiles>CCOC(=O)c1cc(OCC)c(C(=O)OCC)cc1OCC</smiles>

2<smiles>CC#Cc1ccc(B2OC(C)(C)C(C)(C)O2)cc1</smiles>
3 $78 \%$

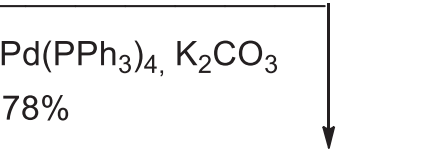<smiles>C#Cc1ccc(-c2cc(C(=O)OCc3ccccc3)c(-c3ccc(C#C)cc3)cc2C(=O)O)cc1</smiles>

1) $\mathrm{K}_{2} \mathrm{CO}_{3}$

2) $\mathrm{KOH}$

3) $\mathrm{HCl}$

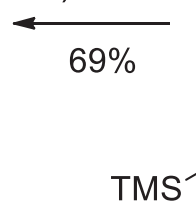

Scheme 1. Synthesis of diyne DETDCA.

with the calcium ions via electrostatics, while the hydroxyl groups form hydrogen bonds with the calcite carbonate group oxygen atoms [23,24]. Furthermore, a detailed investigation towards a possible deprotonation has been performed, suggesting a transition of the hydrogen from the hydroxyl group towards the oxygen of the carbonate group under formation of a hydrogen bond $[23,24]$. Two anchor groups were chosen here for symmetry reasons and to also promote molecule-molecule interactions that can be beneficial for the formation of an ordered selfassembled structure as compared to mobile species.

Our experimental results indicate that DETDCA dimerize in the crucible when choosing a high-temperature deposition route. When following a low-temperature deposition route, the monomers are deposited on the surface. Upon annealing the sample after monomer deposition results in AFM images that can be readily explained by the dimerization of the molecules on the calcite (10.4) surface. Thus, our work provides experimental indication for extending the concept of on-surface homocoupling of terminal alkynes to a bulk insulator surface.

\section{Experimental section}

\subsection{Molecule synthesis}

The synthesis of DETDCA was performed in five steps following the route shown in Scheme 1. First, commercially available diester $\mathbf{1}$ was treated with $\mathrm{Tf}_{2} \mathrm{O}$ to obtain bistriflate 2 in $91 \%$ yield. Then, double Pd-catalyzed Suzuki coupling of bistriflate 2 with two equivalents of boronic ester 3 led to the isolation of diyne 4 in 78\% yield. Finally, sequential treatment of compound 4 with $\mathrm{K}_{2} \mathrm{CO}_{3}, \mathrm{KOH}$ and $\mathrm{HCl}$ afforded DETDCA in 69\% yield (see supporting information for details).

\subsection{Surface preparation and molecule deposition}

Calcite crystals were purchased at Korth Kristalle (Altenholz, Kiel, Germany). Before mounting in the sample holder, the crystallographic directions were identified based on the crystal's birefringence [25]. Prior to molecule deposition, the sample was cleaved in situ and annealed at $625 \mathrm{~K}$. Atomically resolved images were obtained to check the cleanliness of the surface and to confirm the crystallographic directions. The DETDCA molecules were deposited from a home-built Knudsen cell. Two different deposition protocols were used. For the low-temperature deposition route, a crucible temperature of $355 \mathrm{~K}$ and a deposition time of about 12-15 h was used. For the high-temperature deposition route, a crucible temperature of $445 \mathrm{~K}$ and a deposition time of $45 \mathrm{~min}$ to few hours was used.

\subsection{Atomic force microscopy imaging}

For imaging the molecular structures on the bulk insulator surface, we use dynamic AFM imaging operated under UHV conditions. A variable temperature, beam deflection AFM (VT AFM) from ScientaOmicron (Taunusstein, Germany) was used in the so-called frequency modulation mode. Silicon cantilevers from Nanosensors (Neuchâtel, Switzerland) with eigen frequencies in the order of $300 \mathrm{kHz}$ and force constants of about $40 \mathrm{~N} / \mathrm{m}$ were used. The image channels as well as the slow and fast scan directions are given in the upper right corner of the images. During imaging the sample was kept at room temperature. 

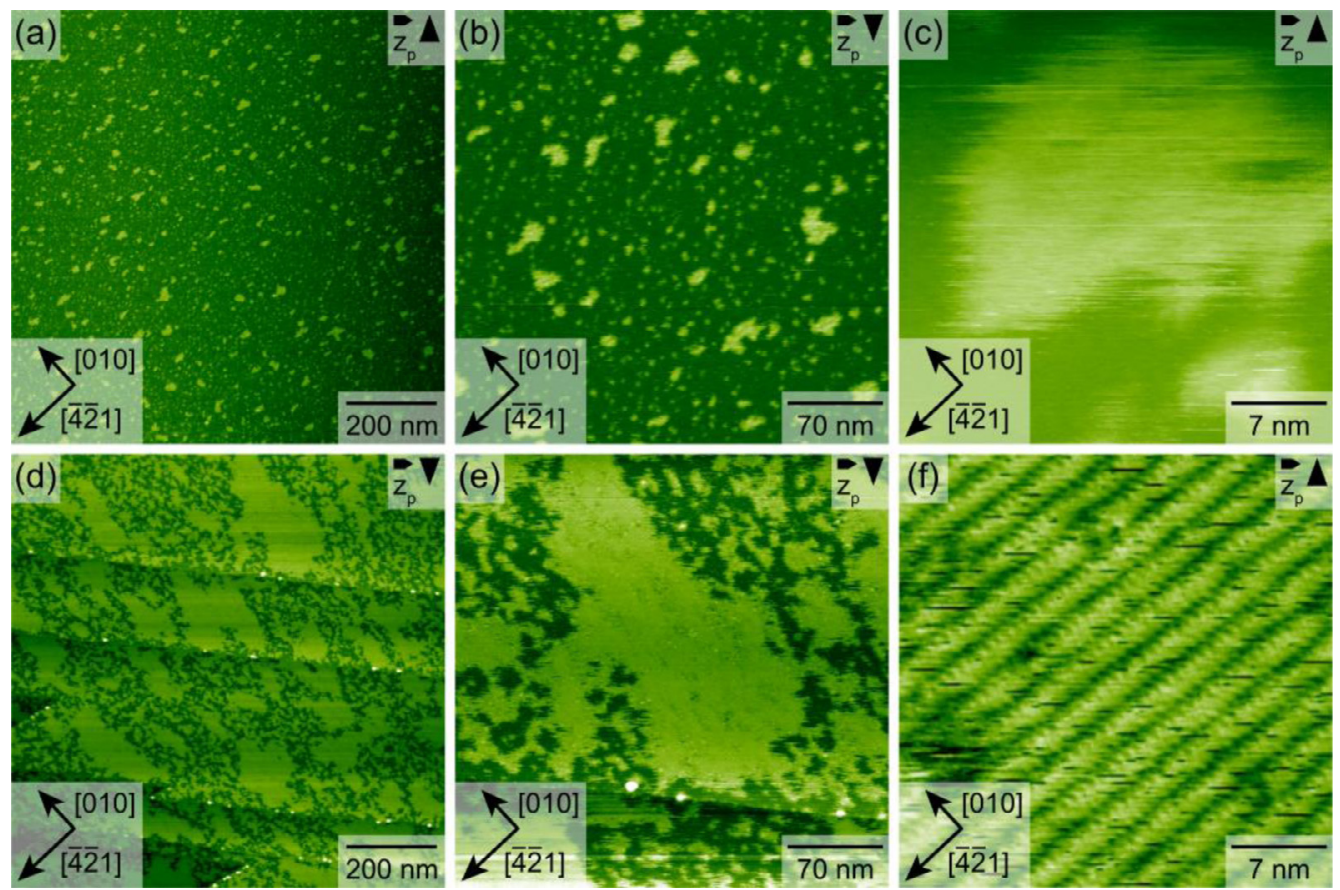

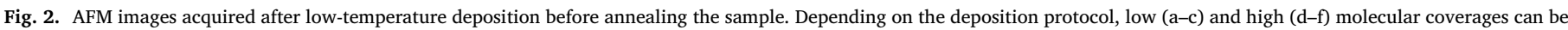
achieved.

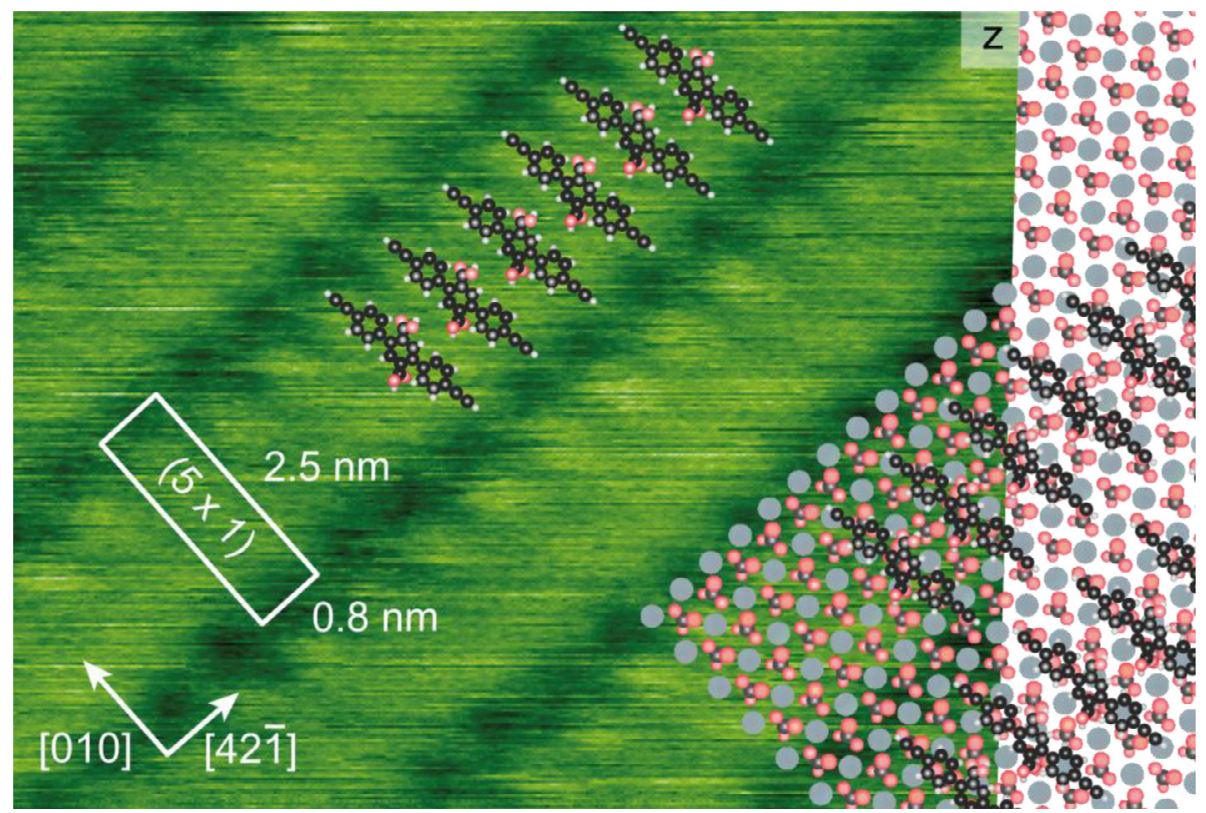

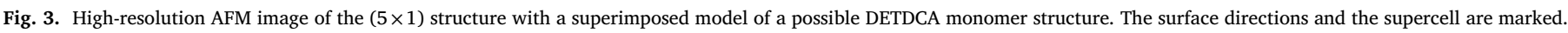

\section{Results and discussion}

\subsection{Low-temperature deposition}

After molecule deposition using the low-temperature deposition protocol (sublimation temperature of about $355 \mathrm{~K}$ for $12-15 \mathrm{~h}$ ), molecular islands are found on the surface as shown in Fig. 2a-c. In the lowcoverage regime, these islands exhibit fuzzy edges, which are indicative of high molecule mobility, resulting in characteristic steaky features. A zoom onto an island in the low-coverage regime (Fig. 2c) does not reveal an inner structure. When increasing the coverage (Fig. 2d-f), the island structures become more stable, which is explained by a reduced molecule mobility. A zoom onto an island in the high-coverage regime unravels a highly ordered inner structure (Fig. 2f).

To further analyze this structure, we performed a high-resolution experiment as shown in Fig. 3 . In this image, the $(5 \times 1)$ superstructure of the molecular island is indicated by a white rectangle. We superimpose a model of the molecules to the image to provide an impression of the molecular dimensions. This superimposed model suggests that the unit cell contains one DETDCA monomer. We also provide a model for the underlying calcite crystal to illustrate the arrangement of the monomers on the calcite surface. We stress, however, while the dimensions and orientation of the molecules are well defined from the experiment, the absolute position of the molecules on the surface is unknown. In the model, 

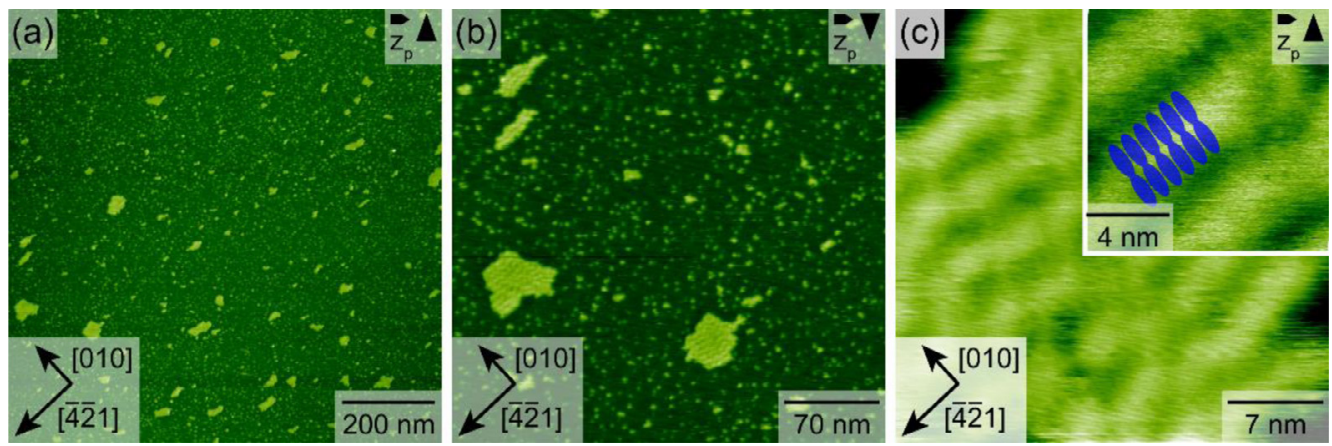

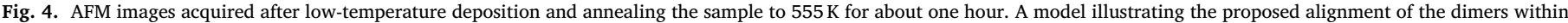
the stripes is given in the inset in (c).<smiles>C#Cc1ccc(-c2cc(C(=O)O)c(-c3ccc(C#CC#Cc4ccc(-c5cc(C(=O)O)c(-c6ccc(C#C)cc6)cc5C(=O)O)cc4)cc3)cc2C(=O)O)cc1</smiles>

Scheme 2. Expected on-surface coupling of DETDCA.

we assume that the molecule is not flat, given that the side groups in the 2-position, namely hydrogen and carboxylic acid group, would overlap. To circumvent this overlap, a rotation of the benzene rings transverse the molecular axes is expected. Hence, only one carboxylic acid group would anchor to the surface while the second one is pointing upwards and perhaps forms a hydrogen bonds with the acid group of the neighboring molecule.

Next, we annealed the sample after low-coverage molecule deposition as shown in Fig. 2a-c to $555 \mathrm{~K}$ for about one hour. The result of such an annealing experiment is shown in Fig. 4. The overall appearance of the islands has remained unchanged upon annealing, as can be seen by comparing the two overview images shown in Figs. 2a and 4a, respectively. However, the inner structure of the islands has changed significantly as demonstrate in the zoom image given in Fig. 4c. After annealing, a stripe-like structure is observed, exhibiting somewhat irregular stripes with a width of about $4.5 \mathrm{~nm}$. This width fits in length with dimerized DETDCA molecules that are linked via homocoupling of the terminal alkynes, as illustrated in the inset in Fig. 4c. Moreover, the island edges appear well-defined and do no longer present the fuzzy structure that was observed for the as-deposited molecular islands. Assuming that the dimers possess a reduced diffusivity on the surface as compared to the monomers, this observation is in line with the above made interpretation of DETDCA dimerization (Scheme 2).

\subsection{High-temperature deposition}

We also performed deposition experiments onto a sample held at room temperature with increased sublimation temperature $(445 \mathrm{~K})$ in an attempted to reduce the long sublimation times needed for the above-mentioned low-temperature deposition protocol. When sublimating with a sublimation temperature of $445 \mathrm{~K}$ for about $15 \mathrm{~h}$, molecular islands as shown in Fig. $5 \mathrm{a}-\mathrm{c}$ are obtained. These islands exhibit the characteristic stripe-like inner structure that was reported above for the annealed sample obtained after the low-temperature deposition protocol. However, a clear difference can be recognized when comparing the island structures in Fig. 4 (low-temperature deposition after annealing) and Fig. 5a-c (high-temperature deposition without annealing). For the high-temperature deposition protocol without annealing, fuzzy edges and streaky features in between the molecular islands are observed, indicative of diffusing molecular species. These high-temperature deposition results can be explained by the direct sublimation of DETDCA dimers, i.e., we suggest that the high sublimation temperature induces dimerization directly in the crucible as has been reported before for 1,4diethynylbenzene [17]. Besides the dimers, few monomers might be deposited simultaneously. These monomers might diffuse in between the island, resulting in the streaky structures observed in the images shown in Fig. 5a-c.

Next, we examined the effect of annealing and irradiating the structures as obtained after high-temperature deposition. We found that both, annealing the sample to about $555 \mathrm{~K}$ and irradiating the sample with a mercury lamp (wavelength $220-590 \mathrm{~nm}$ ), resulted in the same final conditions. This observation agrees with previous results presented in the literature that have demonstrate that coupling of terminal alkynes can be induced by both, annealing or irradiation [19]. The changes induced are illustrated in Fig. 5d-f, which show a sample after irradiation for about $12 \mathrm{~h}$. As can be seen, the inner structure is largely unchanged. However, the edges appear much more well defined and the streaky features in between the islands are vanished. This result indicates that 

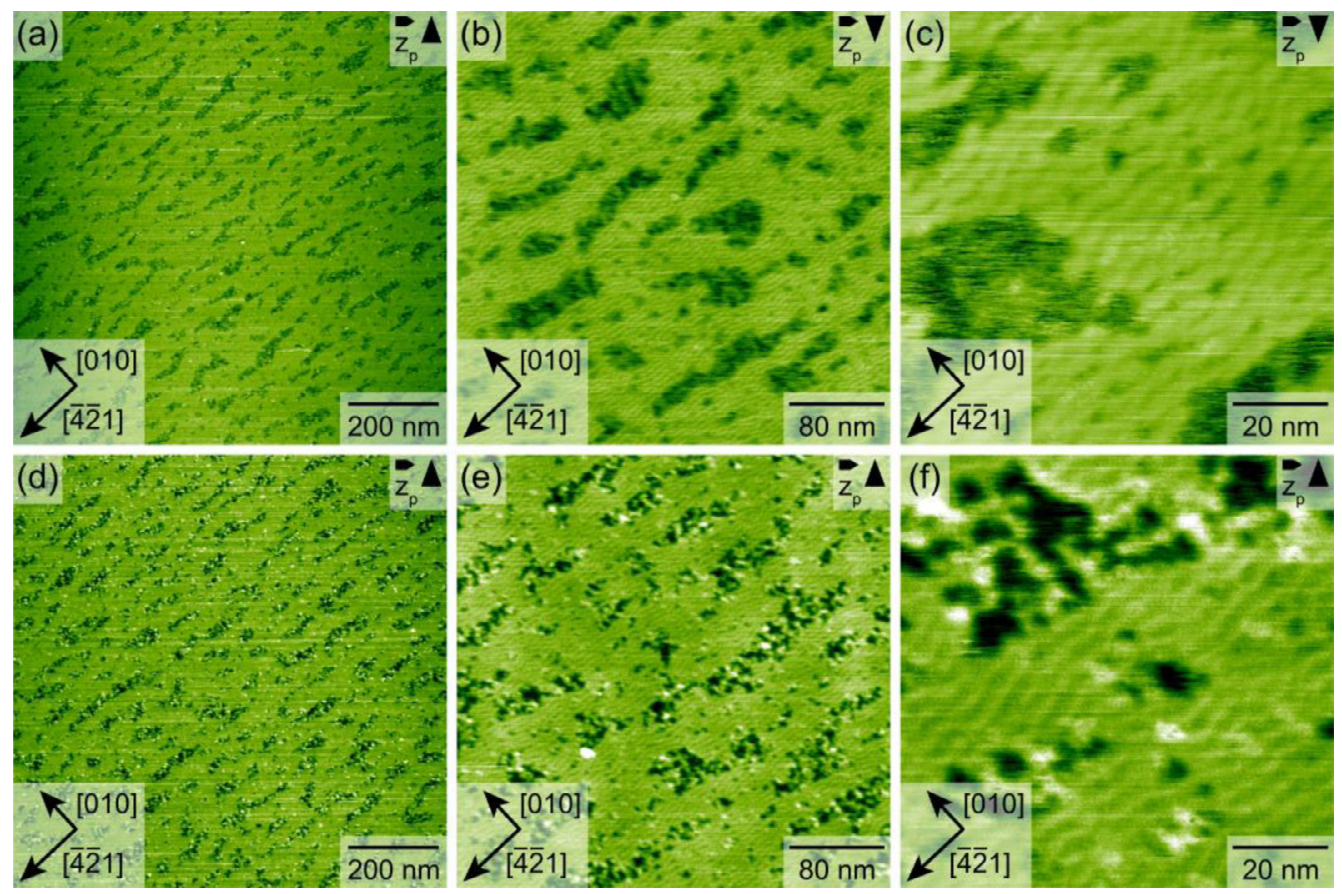

Fig. 5. AFM images acquired after high-temperature deposition before (a-c) and after (d-f) irradiating the sample.

remaining monomers react upon annealing or irradiation, thus, the diffusing species are removed.

To summarize, we suggest that both, annealing to $555 \mathrm{~K}$ and irradiating with mercury lamp (wavelength $220-590 \mathrm{~nm}$ ) can induce homocoupling of the terminal alkynes. The fact that this is possible in the absence of a metal surface indicates that electron transfer from the support surface is not required to induce the reaction. This is in line with a recent theoretical study on coupling of terminal alkynes on $\mathrm{Ag}(111)$ [22]. In the latter study, it was concluded that the surface plays a central role for the reaction in constraining the molecular movement as well as stabilizing reaction intermediates. It is, however, not chemically active in a sense that it, e.g., donates electrons. For the present case, we speculate that the carboxylic acid groups at the molecule core are crucial for anchoring the molecule to the substrate and constrain the mobility on the surface. The limited mobility may furthermore explain why only dimers and no trimers or larger oligomers are formed. This interpretation is in agreement with previous observations in literature on $\mathrm{Ag}(111)$ [19].

\section{Conclusion}

In conclusion, we presented an experimental AFM study investigating the structure formation and reaction of DETDCA molecules on the (10.4) surface of the bulk insulator calcite. When depositing the molecules following a low-temperature deposition route, highly ordered islands are found on the surface that exhibit a $(5 \times 1)$ inner structure. This structure can be readily explained by an ordered arrangement of the DETDCA monomers. The characteristic streaky edges of these islands are explained by diffusing monomer species. Upon annealing, these islands drastically change their structure, as is expected when the monomers dimerize. Now, islands with a striped inner structure are found on the surface. Interestingly, the width of the stripes fits in size with the length of a dimer. Moreover, the fuzzy edges are vanished, giving further evidence for an on-surface dimerization. For the high-temperature deposition protocol, the striped islands are obtained directly, indicative of a direct deposition of the dimers. This result can be easily explained by a dimerization in the crucible, which has been observed before for another diyne molecule. Also here, fuzzy edges vanish after annealing or irradiation, indicative of few remaining monomers that are deposited during the high-temperature route.

\section{Author Contributions}

The manuscript was written through contributions of all authors. All authors have given approval to the final version of the manuscript.

\section{Acknowledgments}

We gratefully acknowledge financial support from the EU through grant PAMS (seventh framework program GA 610446), the Agencia Estatal de Investigación (MAT2016-78293-C6-3-R), the Xunta de Galicia (Centro singular de investigación de Galicia accreditation 2016-2019, ED431G/09) and the European Regional Development Fund (ERDF). We thank Robert Lindner for help in the laboratory.

\section{References}

[1] R.P. Feynman, There's plenty of room at the bottom, Eng. Sci. 23 (1960) 22-36.

[2] S.-W. Hla, L. Bartels, G. Meyer, K.-H. Rieder, Inducing all steps of a chemical reaction with the scanning tunneling microscope tip: towards single molecule engineering, Phys. Rev. Lett. 85 (2000) 2777-2780.

[3] A. Gourdon, On-surface covalent coupling in ultrahigh vacuum, Angew. Chem. Int. Ed. 47 (2008) 6950-6953.

[4] G. Franc, A. Gourdon, Covalent networks through on-surface chemistry in ultra-high vacuum: state-of-the-art and recent developments, Phys. Chem. Chem. Phys. 13 (2011) 14283-14292.

[5] R. Lindner, A. Kühnle, On-surface reactions, ChemPhysChem 16 (2015) 1582-1592.

[6] C. Nacci, S. Hecht, L. Grill, The emergence of covalent on-surface polymerization, in: A. Gourdon (Ed.), Advances in Atom and Single Molecule Machines: On-Surface Synthesis, Springer, Heidelberg, 2016, pp. 1-21.

[7] L. Grill, M. Dyer, L. Lafferentz, M. Persson, M.V. Peters, S. Hecht, Nano-architectures by covalent assembly of molecular building blocks, Nature Nanotech. 2 (2007) $687-691$.

[8] R. Gutzler, H. Walch, G. Eder, S. Kloft, W.M. Heckl, M. Lackinger, Surface mediated synthesis of 2D covalent organic frameworks: 1,3,5-tris(4-bromophenyl)benzene on graphite(001), $\mathrm{Cu}(111)$, and $\mathrm{Ag}(110)$, Chem. Commun. (2009) 4456-4458.

[9] J.M. Cai, P. Ruffieux, R. Jaafar, M. Bieri, T. Braun, S. Blankenburg, M. Muoth, A.P. Seitsonen, M. Saleh, X.L. Feng, K. Müllen, R. Fasel, Atomically precise bottom-up fabrication of graphene nanoribbons, Nature 466 (2010) 470-473.

[10] L. Lafferentz, V. Eberhardt, C. Dri, C. Africh, G. Comelli, F. Esch, S. Hecht, L. Grill, Controlling on-surface polymerization by hierarchical and substrate-directed growth, Nat. Chem. 4 (2012) 215-220.

[11] J. Eichhorn, D. Nieckarz, O. Ochs, D. Samanta, M. Schmittel, P.J. Szabelski, M. Lackinger, On-surface Ullmann coupling: the influence of kinetic reaction pa- 
rameters on the morphology and quality of covalent networks, ACS Nano 8 (2014) 7880-7889.

[12] S. Zint, D. Ebeling, T. Schlöder, S. Ahles, D. Mollenhauer, H.A. Wegner, A. Schirmeisen, Imaging successive intermediate states of the on-surface Ullmann reaction on Cu(111): role of the metal coordination, ACS Nano 11 (2017) 4183-4190.

[13] C. Glaser, Beiträge zur Kenntniss des Acetenylbenzols, Ber. Dtsch. Chem. Ges. 2 (1869) 422-424.

[14] F. Klappenberger, Y.-Q. Zhang, J. Björk, S. Klyatskaya, M. Ruben, J.V. Barth, On-surface synthesis of carbon-based scaffolds and nanomaterials using terminal alkynes, Acc. Chem. Res. 48 (2015).

[15] P. Siemsen, R.C. Livingston, F. Diederich, Acetylenic coupling: a powerful tool in molecular construction, Angew. Chem. Int. Ed. 39 (2000).

[16] Y.-Q. Zhang, N. Kepčija, M. Kleinschrodt, K. Diller, S. Fischer, A.C. Papageorgiou, F. Allegretti, J. Björk, S. Klyatskaya, F. Klappenberger, M. Ruben, J.V. Barth, Homo-coupling of terminal alkynes on a noble metal surface, Nat. Comm. 3 (2012) 1286.

[17] H.-Y.Y. Gao, J.-H.H. Franke, H. Wagner, D. Zhong, P.-A.A. Held, A. Studer, H. Fuchs, Effect of metal surfaces in on-surface Glaser coupling, J. Phys. Chem. C 117 (2013) 18595-18602.

[18] H.-Y.Y. Gao, H. Wagner, D. Zhong, J.-H. Franke, A. Studer, H. Fuchs, Glaser coupling at metal surfaces, Angew. Chem. Int. Ed. 52 (2013) 4024-4028.
[19] H.-Y.Y. Gao, D. Zhong, H. Mönig, H. Wagner, P.-A. Held, A. Timmer, A. Studer, H. Fuchs, Photochemical glaser coupling at metal surfaces, J. Phys. Chem. C 118 (2014) 6272-6277.

[20] J. Eichhorn, W.M. Heckl, M. Lackinger, On-surface polymerization of 1,4-diethynylbenzene on Cu(111), Chem. Commun. 49 (2013) 2900-2902.

[21] B. Cirera, Y.Q. Zhang, J. Björk, S. Klyatskaya, Z. Chen, M. Ruben, J.V. Barth, F. Klappenberger, Synthesis of extended graphdiyne wires by vicinal surface templating, Nano Lett. 14 (2014) 1891-1897.

[22] J. Björk, Y.-Q. Zhang, F. Klappenberger, J.V. Barth, S. Stafström, Unraveling the mechanism of the covalent coupling between terminal alkynes on a noble metal, J. Phys. Chem. C 118 (2014) 3181-3187.

[23] C. Paris, A. Floris, S. Aeschlimann, M. Kittelmann, F. Kling, R. Bechstein, L. Kantorovich, A. Kühnle, Increasing the templating effect on a bulk insulator surface: from a kinetically trapped to a thermodynamically more stable structure, J. Phys. Chem. C 120 (2016) 17546-17554.

[24] A. Richter, V. Haapasilta, C. Venturini, R. Bechstein, A. Gourdon, A.S. Foster, A. Kühnle, Diacetylene polymerization on a bulk insulator surface, Phys. Chem. Chem. Phys. 19 (2017) 15172-15176.

[25] S. Kuhn, M. Kittelmann, Y. Sugimoto, M. Abe, A. Kühnle, P. Rahe, Identifying the absolute orientation of a low-symmetry surface in real space, Phys. Rev. B 90 (2014) 195405. 